\title{
Retarded potentials and the radiative arrow of time
}

\author{
A D Boozer \\ Department of Physics, California Institute of Technology, Pasadena, CA 91125, USA \\ E-mail: boozer@caltech.edu
}

Received 3 July 2007, in final form 14 August 2007

Published 28 September 2007

Online at stacks.iop.org/EJP/28/1131

\begin{abstract}
Maxwell's equations admit both retarded and advanced solutions, but only the retarded solutions seem to characterize the radiative phenomena that actually occur in nature. The reason for this, however, is not usually explained in electrodynamics textbooks. We justify the use of the retarded solutions by considering a simple toy model, which allows us to avoid the technical complexities of electrodynamics and focus on the physical concepts. In addition, we explain how the use of the retarded solutions is related to a number of fundamental issues in physics, such as time-reversal invariance and the radiative arrow of time.
\end{abstract}

\section{Introduction}

Maxwell's equations admit both retarded and advanced solutions, but only the retarded solutions seem to characterize the radiative phenomena that actually occur in nature. The reason for this, however, is not explained in most electrodynamics textbooks; instead, it is simply assumed that the retarded solutions are the only ones that are relevant. In this paper, we justify the use of the retarded solutions by considering a simple toy model, which allows us to avoid the technical complexities of electrodynamics and focus on the physical concepts. In addition, our discussion connects the use of the retarded solutions to a number of fundamental issues in physics, such as time-reversal invariance and the radiative arrow of time. The paper is intended to supplement either a discussion of radiation in an electrodynamics class for advanced undergraduates, or a discussion of the symmetry properties of Maxwell's equations in a class for beginning graduate students.

The fact that radiative phenomena in nature are characterized by the retarded solutions, but not the advanced solutions, means that these phenomena are time asymmetric, and therefore define a preferred orientation, or 'arrow', to time. This is puzzling, because it seems to contradict the fact that Maxwell's equations are time-reversal invariant ${ }^{1}$. There is no real

\footnotetext{
1 The time-reversal invariance of Maxwell's equations is discussed in section 6.11 of [1].
} 
contradiction, however; as in the case of irreversible thermodynamic processes, the time asymmetry is not due to the dynamical laws, but rather to the initial conditions that pertain to the actual world. We will show that because the initial conditions of the world are such as to give rise to time-asymmetric radiative processes, it is useful to formulate electrodynamics in a way that is not time-reversal invariant, and this involves expressing the theory in terms of the retarded solutions rather than the advanced solutions.

The radiative arrow is quite general, and arises not only in electrodynamics but also in many other systems in which waves are radiated. A particularly vivid example can be given for the case of water waves: imagine dropping a stone into a pond of still water ${ }^{2}$. Coherent circular wavefronts will propagate outwards from the point of impact, and will ultimately be absorbed by the sides of the pond. However, such wavefronts never originate at the sides of the pond and propagate inwards, converging on a point in the interior. Thus, the radiative process initiated by the impact of the stone is time asymmetric.

The same issues of time asymmetry and time-reversal invariance that arise in electrodynamics also apply to these other systems, so to clarify these issues we will discuss them in the context of a system that is particularly simple: a toy model of electrodynamics in $(1+1)$ dimensions. This toy model shares much of the conceptual structure of electrodynamics, but is mathematically much simpler.

The paper is organized as follows. In section 2, we briefly describe the toy model, and show how it is analogous to electrodynamics. In section 3, we give two reformulations of the toy model, called the retarded and advanced formulations, which are obtained by expressing the model in terms of retarded and advanced solutions. In section 4, we show that the retarded and advanced formulations are useful for describing scattering processes, and in section 5 we describe two specific scattering processes from the point of view of each of these formulations. Finally, in section 6 , we discuss the time asymmetry of radiation, and show that for initial conditions that describe the actual world it is most natural to use the retarded formulation.

\section{A toy model of electrodynamics}

The toy model that we will be considering describes a coupled particle-field system in $(1+1)$ dimensions, and consists of a scalar potential $\phi(t, x)$ coupled to a single point particle that obeys Newtonian dynamics ${ }^{3}$. The scalar potential $\phi$ is analogous to the vector potential $A^{\mu}$ of electrodynamics, and its derivatives $E \equiv \partial_{x} \phi$ and $B \equiv \partial_{t} \phi$ are analogous to the electric and magnetic fields $\vec{E}$ and $\vec{B}$, respectively. The equations of motion for the $E$ and $B$ fields, the analogs to Maxwell's equations, are ${ }^{4}$

$$
\begin{aligned}
& \partial_{t} E(t, x)=\partial_{x} B(t, x) \\
& \partial_{t} B(t, x)=\partial_{x} E(t, x)-2 g \delta(x-z(t)),
\end{aligned}
$$

where $z$ is the spatial coordinate of the particle and $g$ is its charge. We can use equation (2) to obtain a field equation for the potential $\phi$ by substituting $E=\partial_{x} \phi$ and $B=\partial_{t} \phi$ :

$$
\left(\partial_{t}^{2}-\partial_{x}^{2}\right) \phi(t, x)=-2 g \delta(x-z(t)) .
$$

2 This example is taken from [2].

3 A complete description of the toy model is given in [3]; derivations for the various results that we quote involving the toy model can be found here.

${ }^{4}$ We will choose a system of units such that the speed at which waves are propagated by the field, the analog to the speed of light, is equal to 1 . 
As an example, suppose the particle is stationary at the origin. Using the fact that ${ }^{5}$ $\partial_{x}^{2}|x|=\partial_{x} \epsilon(x)=2 \delta(x)$, we can write the solutions to equation (3) in the form

$$
\phi(t, x)=V(t, x)+\psi(t, x),
$$

where $V(t, x) \equiv g|x|$ and $\psi$ is an arbitrary solution of the homogeneous wave equation

$$
\left(\partial_{t}^{2}-\partial_{x}^{2}\right) \psi(t, x)=0 \text {. }
$$

The potential $V$ describes the static potential produced by the stationary particle, and corresponds to the fields

$$
\begin{aligned}
& E(t, x)=\partial_{x} V(t, x)=g \epsilon(x) \\
& B(t, x)=\partial_{t} V(t, x)=0 .
\end{aligned}
$$

We can think of $V$ and its corresponding fields $E$ and $B$ as the analogs to the electrodynamic Coulomb potential $e / r$ and its corresponding fields $\vec{E}=e \hat{r} / r^{2}$ and $\vec{B}=0$. The potential $\psi$ describes freely propagating waves ${ }^{6}$, or radiation, which can be added to this static potential. Note that $\psi$ is not uniquely determined by the field equation (3), rather it is set by the initial conditions for the system.

We have described the fields in the toy model; let us now consider the particle. We will assume that the particle is harmonically bound at the origin, and that the $E$ field exerts a force on the particle that is given by

$$
F_{f}(t)=-2 g E(t, z(t)) \text {. }
$$

This is analogous to the Lorentz force of electrodynamics. The state of the particle is determined by its position $z$ and momentum $p$, and the equations of motion for these quantities are

$$
\begin{aligned}
& \dot{z}=p / m \\
& \dot{p}=-m \omega_{0}^{2} z+F_{f}=-m \omega_{0}^{2} z-2 g E(t, z(t)),
\end{aligned}
$$

where $\omega_{0}$ is the harmonic frequency for the binding potential.

The equations of motion (9), (10) for the particle and (1), (2) for the fields fully describe the coupled particle-field system. At a given time $t$, the state of the system is completely determined by specifying the particle variables $z(t)$ and $p(t)$, together with the field variables $E(t, x)$ and $B(t, x)$ at all points in space. By integrating the equations of motion, we can evolve this state in time; let us describe this time-evolution process in terms of an operator $\mathcal{E}_{\tau}$, defined such that

$\mathcal{E}_{\tau}\{z(t), p(t), E(t, x), B(t, x)\}=\{z(t+\tau), p(t+\tau), E(t+\tau, x), B(t+\tau, x)\}$.

The evolution given by $\mathcal{E}_{\tau}$ has an important property: it is invariant under time reversal ${ }^{7}$. By this we mean the following. Let us define a motion-reversal operator $\mathcal{R}$ that acts on states by flipping the sign of $p(t)$ and $B(t, x)$ :

$$
\mathcal{R}\{z(t), p(t), E(t, x), B(t, x)\}=\{z(t),-p(t), E(t, x),-B(t, x)\} .
$$

When we say that the evolution is time-reversal invariant, we mean that reversing the motion and then evolving the system forward in time is equivalent to evolving the system backward in time and then reversing the motion: $\mathcal{E}_{\tau} \circ \mathcal{R}=\mathcal{R} \circ \mathcal{E}_{-\tau}$.

5 The following notation is used in this paper: $\theta(x)$ is the step function, defined such that $\theta(x)=1$ for $x>0, \theta(x)=1 / 2$ for $x=0, \theta(x)=0$ for $x<0 ; \epsilon(x)$ is the sign function, defined such that $\epsilon(x)=1$ for $x>0, \epsilon(x)=0$ for $x=0, \epsilon(x)=-1$ for $x<0$.

6 In principle, $\psi$ could also contain the terms $E_{0} x$ and $B_{0} t$, which describe constant background fields rather than waves, but we will not consider such terms here.

7 This notion of time-reversal invariance is discussed in section 4.4 of [4] and the time-reversal invariance of Maxwell's equations is discussed in section 6.11 of [1]. 


\section{Retarded and advanced formulations of the toy model}

We can think of the potential $\phi$ as consisting of two components: one that is produced by the particle and one that describes freely propagating radiation. We saw this in the example from the previous section involving a stationary particle, where we expressed $\phi$ as the sum of a static potential $V$ and a radiation potential $\psi$. It is useful to reformulate the toy model by decomposing $\phi$ so as to separate out these two components. In this section, we describe two such formulations, based on two different decompositions of $\phi$, and in the following section we show that these formulations are useful for describing scattering processes.

In the first formulation, which we will call the retarded formulation, we decompose the total potential $\phi$ into a retarded potential $\phi_{r}$ and an in potential $\phi_{\text {in }}$ :

$$
\phi=\phi_{r}+\phi_{\text {in }} .
$$

The retarded potential is analogous to the Liénard-Wiechert potentials of electrodynamics ${ }^{8}$. It describes the potential produced by the particle and is defined by

$$
\phi_{r}(t, x)=-g \int_{T_{r}}^{\infty} \theta\left(t-t^{\prime}-\left|x-z\left(t^{\prime}\right)\right|\right) \mathrm{d} t^{\prime}+g\left(t-T_{r}\right),
$$

where $T_{r}$ is a constant. One can show that the retarded potential is a solution to the inhomogeneous wave equation

$$
\left(\partial_{t}^{2}-\partial_{x}^{2}\right) \phi_{r}(t, x)=-2 g \delta(x-z(t)),
$$

and that in the low-velocity limit the retarded fields are given by ${ }^{9}$

$$
\begin{aligned}
& E_{r}(t, x)=\partial_{x} \phi_{r}(t, x)=g \epsilon(x-z(t))+g v\left(t_{r}(t, x)\right) \\
& B_{r}(t, x)=\partial_{t} \phi_{r}(t, x)=-g v\left(t_{r}(t, x)\right) \epsilon(x-z(t)) .
\end{aligned}
$$

In these expressions $v\left(t_{r}(t, x)\right)$ is the velocity of the particle at the retarded time $t_{r}(t, x)$, which is defined implicitly by the equation

$$
t_{r}=t-\left|x-z\left(t_{r}\right)\right| .
$$

Note that if the particle is stationary at the origin, then $v\left(t_{r}(t, x)\right)=0$ and the retarded fields given in equations (16) and (17) are just the static fields given in equations (6) and (7).

Because both the total potential $\phi$ and the retarded potential $\phi_{r}$ are solutions to the inhomogeneous wave equation, it follows from equation (13) that $\phi_{\text {in }}$ is a solution to the homogeneous wave equation

$$
\left(\partial_{t}^{2}-\partial_{x}^{2}\right) \phi_{\text {in }}(t, x)=0,
$$

and therefore describes freely propagating radiation. From equation (19), we can obtain the equations of motion for the corresponding fields $E_{\text {in }} \equiv \partial_{x} \phi_{\text {in }}$ and $B_{\text {in }} \equiv \partial_{t} \phi_{\text {in }}$ :

$$
\begin{aligned}
& \partial_{t} E_{\text {in }}(t, x)=\partial_{x} B_{\text {in }}(t, x) \\
& \partial_{t} B_{\text {in }}(t, x)=\partial_{x} E_{\text {in }}(t, x) .
\end{aligned}
$$

We can use the decomposition of $\phi$ given in equation (13) to decompose the force $F_{f}$ given in equation (8) into a component $F_{\text {in }}$ due to the in field and a component $F_{r}$ due to the

8 The Liénard-Wiechert potentials of electrodynamics are derived in section 9.2 of [5].

9 These expressions hold everywhere except on the worldline of the particle itself; that is, they hold for all events $(t, x)$ such that $x \neq z(t)$. 
retarded field: $F_{f}=F_{\text {in }}+F_{r}$. One can show that $F_{r}$ describes the radiation damping force that the particle exerts on itself, and is given by

$$
F_{r}=-\gamma p /\left(1-v^{2}\right)
$$

where $\gamma \equiv 2 g^{2} / m$. Using these results, we see that in the low-velocity limit we can rewrite the particle equations of motion (9) and (10) as

$$
\begin{aligned}
& \dot{z}=p / m \\
& \dot{p}=-m \omega_{0}^{2} z-\gamma p+F_{\text {in }}=-m \omega_{0}^{2} z-\gamma p-2 g E_{\text {in }}(t, z(t)) .
\end{aligned}
$$

Equations (23) and (24) can be combined to give

$$
\ddot{z}+\gamma \dot{z}+\omega_{0}^{2} z=\frac{1}{m} F_{\text {in }} .
$$

This is analogous to the Abraham-Lorentz equation of motion for electrodynamics ${ }^{10}$.

Let us summarize what we have accomplished. In the original formulation, the state of the system is specified by the particle variables $z, p$ and the field variables $E, B$, which evolve in time according to the equations of motion (1), (2), (9), (10). In the retarded formulation, the state of the system is specified by the particle variables $z, p$ and the field variables $E_{\text {in }}, B_{\text {in }}$, which evolve in time according to the equations of motion (20), (21), (23), (24). In exchanging the old field variables $E, B$ for the new field variables $E_{\text {in }}, B_{\text {in }}$, we have removed a component of the field that is produced by the particle, leaving a component that describes freely propagating radiation.

We can obtain a second formulation, which we will call the advanced formulation, by decomposing the total potential $\phi$ into an advanced potential $\phi_{a}$ and an out potential $\phi_{\text {out }}$ :

$$
\phi=\phi_{a}+\phi_{\text {out }} .
$$

The advanced formulation is entirely analogous to the retarded formulation: the advanced potential corresponds to the retarded potential, and the out potential corresponds to the in potential. The advanced potential is defined by

$$
\phi_{a}(t, x)=-g \int_{-\infty}^{T_{a}} \theta\left(t^{\prime}-t-\left|x-z\left(t^{\prime}\right)\right|\right) \mathrm{d} t^{\prime}+g\left(T_{a}-t\right),
$$

where $T_{a}$ is a constant. In the low-velocity limit, the advanced fields are

$$
\begin{aligned}
& E_{a}(t, x)=\partial_{x} \phi_{a}(t, x)=g \epsilon(x-z(t))-g v\left(t_{a}(t, x)\right) \\
& B_{a}(t, x)=\partial_{t} \phi_{a}(t, x)=-g v\left(t_{a}(t, x)\right) \epsilon(x-z(t)),
\end{aligned}
$$

where $t_{a}(t, x)$, the advanced time corresponding to the event $(t, x)$, is defined implicitly by the equation

$$
t_{a}=t+\left|x-z\left(t_{a}\right)\right|
$$

The out potential satisfies the homogeneous wave equation, and the equations of motion for the corresponding fields are

$$
\begin{aligned}
& \partial_{t} E_{\text {out }}(t, x)=\partial_{x} B_{\text {out }}(t, x) \\
& \partial_{t} B_{\text {out }}(t, x)=\partial_{x} E_{\text {out }}(t, x) .
\end{aligned}
$$

${ }^{10}$ The Abraham-Lorentz equation of motion is discussed in section 17.2 of [1]. 
If we decompose the force $F_{f}$ into components $F_{a}$ and $F_{\text {out }}$, and use the fact that

$$
F_{a}=\gamma p /\left(1-v^{2}\right),
$$

we can write the particle equations of motion as

$$
\begin{aligned}
& \dot{z}=p / m \\
& \dot{p}=-m \omega_{0}^{2} z+\gamma p+F_{\text {out }}=-m \omega_{0}^{2} z+\gamma p-2 g E_{\text {out }}(t, z(t)) .
\end{aligned}
$$

Thus, in the advanced formulation the state of the system is specified by $z, p, E_{\text {out }}, B_{\text {out }}$, and these variables evolve in time according to the equations of motion (31), (32), (34) and (35).

Although the original theory is invariant under time reversal, neither the retarded nor the advanced formulation of the theory shares this property. For example, in the retarded formulation, the state of the system is given by

$$
\left\{z(t), p(t), E_{\text {in }}(t, x), B_{\text {in }}(t, x)\right\} .
$$

As before, we can define a time-evolution operator $\mathcal{E}_{\tau}^{r}$ that evolves these states in time under the equations of motion (20), (21), (23), (24), and we can define a motion-reversal operator $\mathcal{R}^{r}$ that acts on these states by flipping the sign of $p(t)$ and $B_{\text {in }}(t, x)$. Because of the damping term in the equation (24), evolving the system in time and then reversing the motion is not equivalent to reversing the motion and then evolving the system in time: $\mathcal{E}_{\tau}^{r} \circ \mathcal{R}^{r} \neq \mathcal{R}^{r} \circ \mathcal{E}_{-\tau}^{r}$. This is to be expected: the retarded and advanced fields each pick out a particular direction of time, so the decompositions given in equations (13) and (26) are time asymmetric, and the formulations that result from them are not time-reversal invariant ${ }^{11}$.

\section{Scattering and asymptotic conditions}

Consider a process in which a pulse of radiation approaches the particle and scatters off it. We will assume that long before and long after the scattering event, the particle is stationary at the origin, and is spatially separated from any radiation that is present ${ }^{12}$. The value of the retarded and advanced formulations is that they are useful for describing scattering processes in which these asymptotic conditions are satisfied ${ }^{13}$.

The advantage of the retarded formulation is that it provides a simple description of the system before the scattering event occurs. At this time the particle is at rest, so $v\left(t_{r}(t, x)\right)=0$, and the retarded fields are just the static fields produced by the stationary particle. Thus, before the scattering event the in fields describe any incoming radiation that is present, which gets added to the static field to give the total field. After the scattering event, however, the description is more complicated: the retarded field describes both the static field of the particle and the radiation it produced during the scattering event, while the in field describes what would have happened to the incoming radiation if it had not interacted with the particle and had instead continued to propagate freely.

In the retarded formulation it is easy to extrapolate from the state of the system before the scattering event to the state of the system at all times. To see how this works, suppose that at some time $t_{i}$ before the scattering event the state of the system is $\left\{z\left(t_{i}\right)=0, p\left(t_{i}\right)=0, E\left(t_{i}, z\right), B\left(t_{i}, x\right)\right\}$. At time $t_{i}$ the retarded fields are just the static fields

\footnotetext{
11 The retarded and advanced formulations are not the only ways in which the toy model can be reformulated; in general, one could write $\phi=\phi_{r a}+\phi_{i o}$, where $\phi_{r a} \equiv x \phi_{r}+(1-x) \phi_{a}$ and $\phi_{i o} \equiv x \phi_{\text {in }}+(1-x) \phi_{\text {out }}$. The retarded and advanced formulations correspond to the choices $x=1$ and $x=0$.

12 In the toy model a moving particle radiates, so if the particle is spatially separated from the radiation it must be stationary.

${ }^{13}$ The role of asymptotic conditions in electrodynamics is discussed in section 6.4 of [6].
} 
of the particle, which we can subtract from $E\left(t_{i}, z\right)$ and $B\left(t_{i}, x\right)$ to obtain initial conditions for the in fields:

$$
\begin{aligned}
& E_{\text {in }}\left(t_{i}, x\right)=E\left(t_{i}, x\right)-g \epsilon(x) \\
& B_{\text {in }}\left(t_{i}, x\right)=B\left(t_{i}, x\right) .
\end{aligned}
$$

Using these initial conditions, we can immediately write the $i n$ fields at all times:

$$
\begin{aligned}
& E_{\text {in }}(t, x)=\frac{1}{2}\left[E_{\text {in }}\left(t_{i}, x+\tau\right)+E_{\text {in }}\left(t_{i}, x-\tau\right)+B_{\text {in }}\left(t_{i}, x+\tau\right)-B_{\text {in }}\left(t_{i}, x-\tau\right)\right] \\
& B_{\text {in }}(t, x)=\frac{1}{2}\left[B_{\text {in }}\left(t_{i}, x+\tau\right)+B_{\text {in }}\left(t_{i}, x-\tau\right)+E_{\text {in }}\left(t_{i}, x+\tau\right)-E_{\text {in }}\left(t_{i}, x-\tau\right)\right],
\end{aligned}
$$

where $\tau \equiv t-t_{i}$. It is straightforward to verify these expressions by substituting them into the equations of motion (20) and (21) for the in fields. Using equation (39) for $E_{\text {in }}(t, x)$, we can integrate the particle equations of motion (23) and (24) subject to the initial conditions $z\left(t_{i}\right)=p\left(t_{i}\right)=0$ to obtain the particle trajectory $(z(t), p(t))$. Thus, we obtain the state of the system at all times.

Whereas the retarded formulation gives a simple description of the system before the scattering event and a complicated description afterwards, just the opposite is true of the advanced formulation: it gives a simple description of the system after the scattering event and a complicated description before. After the scattering event the particle is once again stationary, so $v\left(t_{a}(t, x)\right)=0$ and the advanced fields are just the static fields of the stationary particle. Thus, after the scattering event the out fields describe the outgoing radiation, which includes both the radiation produced by the particle during the scattering event and the unscattered portion of the incoming radiation.

Using the advanced formulation, one can easily extrapolate from the state of the system after the scattering event to the state of the system at all times. We will not go through the details, but this closely parallels the procedure we described for the retarded formulation: given a final state, one subtracts the static field of the particle to obtain final conditions for the out fields. These final conditions can be used to obtain the out fields at all times, which in turn can be used to evolve the particle backwards in time using the equations of motion (34) and (35).

\section{Examples of scattering processes}

\subsection{Incoming pulse of radiation}

To help make these issues more concrete, we will describe a specific scattering process from the point of view of both the retarded and advanced formulations. We will take the incoming pulse of radiation to have the form

$$
\phi_{\text {in }}(t, x)=\operatorname{Re}(\mathrm{i} / \omega) f(t-x),
$$

where

$$
f(\tau)=A \mathrm{e}^{-\Gamma|\tau|-\mathrm{i} \omega \tau} .
$$

This describes a rightwardly-propagating wavepacket that has centre frequency $\omega$ and spatial extent $\sim 1 / \Gamma$. For simplicity, we will make several assumptions. First, we will assume that the damping constant $\gamma$ is small compared with the harmonic frequency $\omega_{0}$ of the binding potential $\left(\gamma \ll \omega_{0}\right)$. Next, we will assume that the wavepacket is very broad $(\Gamma \ll \gamma)$, and nearly resonant with the particle $\left(\delta \ll \omega_{0}\right.$, where $\left.\delta \equiv \omega-\omega_{0}\right)$. Also, we will work in the 
dipole approximation, which means we assume that the amplitude of the incoming radiation is small enough that the displacement of the particle is always much less than the wavelength of the radiation $(\omega z \ll 1)$. As we shall see, the dipole approximation implies that the particle is slowly moving $(v \ll 1)$, so a low-velocity approximation is justified.

We want to calculate the trajectory of the particle, so we need to determine the force that the in field exerts on it. From equation (41), we find that the in fields are

$$
\begin{aligned}
& E_{\text {in }}(t, x)=\partial_{x} \phi_{\text {in }}(t, x)=-\operatorname{Re} f(t-x) \\
& B_{\text {in }}(t, x)=\partial_{t} \phi_{\text {in }}(t, x)=\operatorname{Re} f(t-x) .
\end{aligned}
$$

Thus, the force that the in field exerts on the particle is

$$
F_{\text {in }}(t)=-2 g E_{\text {in }}(t, z(t))=2 g A \mathrm{e}^{-\Gamma|t-z(t)|} \cos \omega(t-z(t)) .
$$

Because we are working in the dipole approximation, $F_{\text {in }}$ can be approximated as

$$
F_{\text {in }}(t)=2 g A \mathrm{e}^{-\Gamma|t|} \cos \omega t .
$$

We can substitute this expression for $F_{\text {in }}$ into the equation of motion (25) for the particle. Because $F_{\text {in }}$ is now an explicit function of time, and does not depend on the particle position $z(t)$, we can express the solution as ${ }^{14}$

$$
z(t)=\int G_{r}\left(t-t^{\prime}\right) F_{\text {in }}\left(t^{\prime}\right) \mathrm{d} t^{\prime},
$$

where

$$
G_{r}(t) \equiv \frac{1}{m \beta} \theta(t) \mathrm{e}^{-\gamma t / 2} \sin \beta t
$$

and $\beta \equiv\left(\omega_{0}^{2}-\gamma^{2} / 4\right)^{1 / 2}$. It is straightforward to evaluate equation (47) in the regime $\Gamma \ll \gamma \ll \omega_{0}$ in which we are working; the result is

$$
z(t)=-\left(1 / g \omega_{0}\right) \operatorname{Im} \rho(\delta) f(t)
$$

where we have defined

$$
\rho(\delta) \equiv(1-2 \mathrm{i} \delta / \gamma)^{-1}
$$

The velocity of the particle is

$$
v(t)=\dot{z}(t)=(1 / g) \operatorname{Re} \rho(\delta) f(t) .
$$

Because we are working in the dipole approximation, we can approximate the retarded and advanced times by

$$
\begin{aligned}
& t_{r}(t, x) \simeq t-|x| \\
& t_{a}(t, x) \simeq t+|x| .
\end{aligned}
$$

If we combine these results with the expression for the particle velocity given in equation (51), we obtain expressions for the velocity at the retarded and advanced times. Substituting $v\left(t_{r}(t, x)\right)$ into equations (16) and (17), we find that the retarded fields are

$$
\begin{aligned}
& E_{r}(t, x)=g \epsilon(x)+\operatorname{Re} \rho(\delta) f(t-|x|) \\
& B_{r}(t, x)=-\operatorname{Re} \rho(\delta) f(t-|x|) \epsilon(x) .
\end{aligned}
$$

${ }^{14}$ This follows from the fact that $z(t)=G_{r}(t)$ is a solution to equation (25) for $F_{\text {in }}(t)=\delta(t)$ (see section 3.10 of [7]). 


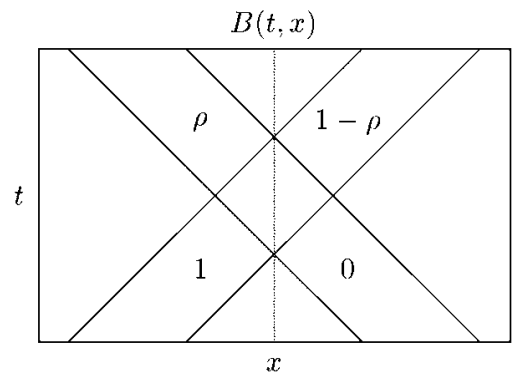

Figure 1. The total field $B(t, x)$ : an incoming pulse $f(t-x)$ approaches the particle from the left and scatters off it, resulting in outgoing pulses $\rho(\delta) f(t+x)$ and $(1-\rho(\delta)) f(t-x)$. The dotted line indicates the trajectory of the particle.
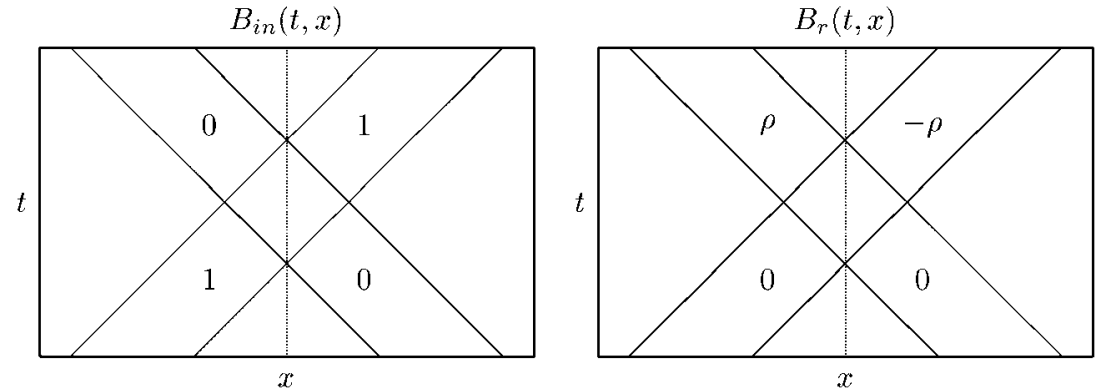

Figure 2. The total field $B(t, x)$ can be decomposed into an $i n$ field $B_{\text {in }}(t, x)$, which consists of a pulse $f(t-x)$ that freely propagates to the right, and a retarded field $B_{r}(t, x)$, which consists of outgoing pulses $\rho(\delta) f(t+x)$ and $-\rho(\delta) f(t-x)$. The dotted line indicates the trajectory of the particle.

Substituting $v\left(t_{a}(t, x)\right)$ into equations (28) and (29), we find that the advanced fields are

$$
\begin{aligned}
& E_{a}(t, x)=g \epsilon(x)-\operatorname{Re} \rho(\delta) f(t+|x|) \\
& B_{a}(t, x)=-\operatorname{Re} \rho(\delta) f(t+|x|) \epsilon(x) .
\end{aligned}
$$

The total fields $E(t, x)$ and $B(t, x)$ are given by the sum of the $i n$ fields and the retarded fields:

$$
\begin{aligned}
& E(t, x)=g \epsilon(x)-\operatorname{Re}[f(t-x)-\rho(\delta) f(t-|x|)] \\
& B(t, x)=\operatorname{Re}[f(t-x)-\rho(\delta) f(t-|x|) \epsilon(x)] .
\end{aligned}
$$

We can obtain the out fields by subtracting the advanced fields from the total fields:

$$
\begin{aligned}
& E_{\text {out }}(t, x)=\operatorname{Re}[\rho(\delta) f(t+x)-(1-\rho(\delta)) f(t-x)] \\
& B_{\text {out }}(t, x)=\operatorname{Re}[\rho(\delta) f(t+x)+(1-\rho(\delta)) f(t-x)] .
\end{aligned}
$$

The total field $B(t, x)$ is schematically illustrated in figure 1. Its decomposition into an $i n$ field and a retarded field is shown in figure 2, and its decomposition into an out field and an advanced field is shown in figure 3 . 

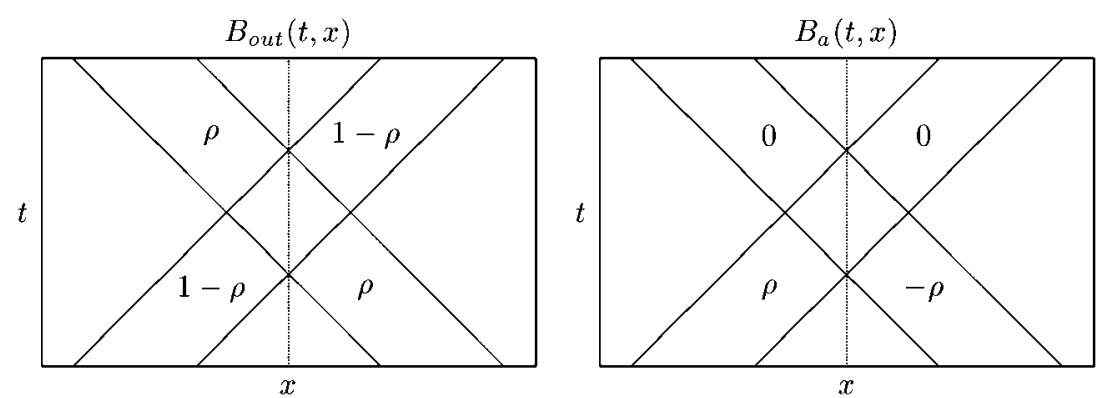

Figure 3. The total field $B(t, x)$ can also be decomposed into an out field $B_{\text {out }}(t, x)$, which consists of pulses $(1-\rho(\delta)) f(t-x)$ and $\rho(\delta) f(t+x)$ freely propagating to the right and left, and an advanced field $B_{a}(t, x)$, which consists of incoming pulses $\rho(\delta) f(t-x)$ and $-\rho(\delta) f(t+x)$. The dotted line indicates the trajectory of the particle.

\subsection{External driving force}

As a second example, let us consider a process in which there is no incoming radiation and the particle is driven by an externally applied force $F_{\text {ext }}$. It is straightforward to modify the equations of motion for the particle to accommodate such a force; we need only add $F_{\text {ext }}$ to the equation of motion for the particle momentum $p$. For simplicity, we will assume that $F_{\text {ext }}$ has the same form as the force $F_{\text {in }}$ given in equation (46):

$$
F_{\text {ext }}(t)=2 g A \mathrm{e}^{-\Gamma|t|} \cos \omega t
$$

This allows us to carry over several of the results from the previous example; specifically, the particle trajectory is given by equation (49), the retarded fields are given by (54), (55) and the advanced fields are given by (56) and (57). We have stipulated that there is no incoming radiation $\left(E_{\text {in }}=B_{\text {in }}=0\right)$, so the total fields are just the retarded fields $\left(E=E_{r}, B=B_{r}\right)$ and the out fields are given by

$$
\begin{aligned}
& E_{\text {out }}(t, x)=E_{r}(t, x)-E_{a}(t, x)=\operatorname{Re} \rho(\delta)(f(t+x)+f(t-x)) \\
& B_{\text {out }}(t, x)=B_{r}(t, x)-B_{a}(t, x)=\operatorname{Re} \rho(\delta)(f(t+x)-f(t-x)) .
\end{aligned}
$$

\section{Time asymmetry}

We have described a toy model that is time-reversal invariant, and shown that it can be reformulated in ways that are not time-reversal invariant by expressing it in terms of either the retarded or the advanced solutions. These reformulations are useful, because they give a simple description of radiative processes that satisfy certain asymptotic conditions. We will now show how these results are related to the time asymmetry of radiation.

Let us begin by generalizing the pond example given in the introduction to other systems in which waves are radiated. For such systems, let us define an emitter to be something that transfers energy to the wave field, and an absorber to be something that transfers energy from the wave field. We will say that an emitter is coherent if after the energy transfer has taken place the wave field is organized into coherent wavefronts diverging from the emitter, and that an absorber is coherent if before the energy transfer has taken place the wave field is organized into coherent wavefronts converging on the absorber. The general observation, which applies to light waves, sound waves, water waves and other radiating systems, is that 
emitters are coherent (e.g. radio transmitters, loudspeakers and stones dropping into ponds), while absorbers are not coherent (e.g. radio receivers, ears and the sides of ponds). Because a coherent absorber is just the time reverse of a coherent emitter, this observation implies that radiative processes are time asymmetric.

It is instructive to compare this radiative time asymmetry with the time asymmetry of thermodynamics. Thermodynamics describes the behaviour of macroscopic systems that are made up of a large number of microscopic constituents, which evolve in time according to a set of dynamical laws. Despite the fact that these dynamical laws are generally timereversal invariant, many thermodynamic processes are time asymmetric; for example, consider a thermodynamic process in which two objects are brought into thermal contact. If the objects are initially at different temperatures then heat will flow from the hot object to the cold object until the temperatures equilibrate; however, if the objects are initially at the same temperature, heat will never spontaneously flow from one object to the other. We can compare this thermodynamic process to the radiative process in which a stone is dropped into a pond and note two points of similarity. First, both the thermodynamic process and the radiative process are irreversible, in the sense that although the time reverse of these processes do not violate the dynamical laws, the initial conditions that pertain to the actual world are such that the time-reversed processes never occur in nature ${ }^{15}$. Second, for both radiation and for thermodynamics, the fact that these initial conditions give rise to irreversible processes implies that they are highly unusual: if one were to randomly pick initial conditions from phase space, using the natural probability measure in which equal phase-space volumes are equally likely to be picked, it is very unlikely that they would describe a world in which irreversible processes occurred.

In the case of thermodynamics, it is overwhelmingly likely that randomly picked initial conditions would describe a world that is near thermal equilibrium and has close to the maximum possible entropy. If these initial conditions were evolved in time, forwards or backwards, the world would not significantly deviate from thermal equilibrium, and the entropy would undergo small fluctuations while remaining near the maximum possible value; thus, there would be no irreversible processes ${ }^{16}$. The actual world, however, is not like this: it evolved from a low-entropy initial state that was very far from thermal equilibrium. The natural tendency of this statistically unlikely initial state to evolve into a statistically likely state of thermal equilibrium gives rise to thermodynamic processes that are irreversible.

In the case of radiation, it is overwhelmingly likely that for randomly picked initial conditions the energy of the system would be spread throughout space and distributed across all the modes of the field. There would be no coherent waves at all, and hence there would be neither coherent emitters nor coherent absorbers. Thus, if we randomly picked initial conditions for the toy model, we could not define an asymptotic limit in which the radiation and the particle are spatially separated, and the retarded and advanced formulations would not be useful. Radiation would be in constant contact with the particle and would act as a heat bath, driving the particle with a randomly fluctuating force. If we were to evolve these initial conditions in time, forwards or backwards, the fluctuating force would cause the particle to exchange energy with the radiation field, but no coherent waves would be emitted or absorbed; thus, there would be no irreversible radiative processes.

\footnotetext{
${ }^{15}$ Note, however, that the problem of explaining these processes in terms of the underlying dynamical laws is far more complicated for the thermodynamic process than for the radiative process. In the case of the thermodynamic process, one needs to connect the dynamical laws, which pertain to the microscopic constituents of the system, to the system's macroscopic behaviour. This is a difficult problem, which does not arise in the case of the radiative process. ${ }^{16}$ It is possible for the system to undergo a large fluctuation in which the entropy deviates significantly from the maximum possible value, but one would need to evolve the system for an extremely long time before such a fluctuation would occur (the timescales involved are of the order of the Poincaré cycle time (see section 4.5 of [8])).
} 
Again, the actual world is not like this. In many systems the field is mostly in a vacuum state, together with some localized packets of radiation. As we saw in the toy model, for this type of state the asymptotic conditions are satisfied, and it is convenient to use either the retarded or advanced formulations, depending on whether we want to predict the future of a scattering process based on an initial state, or retrodict the past of a scattering process based on its final state. Usually we are more interested in prediction than retrodiction, so we choose the retarded rather than the advanced formulation.

There is an additional reason for choosing the retarded formulation, however, which reflects an objective asymmetry in the world, namely the time asymmetry of radiative processes in nature. In the toy model, the analogs to the radiative processes that could occur in nature are those for which the particle is a coherent emitter, but not a coherent absorber. Let us call processes that meet these requirements 'natural processes'. For natural processes the total potential $\phi$ is more closely matched by the retarded potential than by the advanced potential, because the retarded potential describes outgoing radiation that is emitted by the particle, while the advanced potential describes incoming radiation that is absorbed by the particle. Thus, subtracting the retarded potential $\phi_{r}$ from the total potential $\phi$ removes the outgoing coherent wavefronts centred on the particle, leaving a simple expression for $\phi_{\text {in }}=\phi-\phi_{r}$. Although we can also subtract the advanced potential $\phi_{a}$ from the total potential, this leaves a complicated expression for $\phi_{\text {out }}=\phi-\phi_{a}$ : whereas for $\phi_{\text {in }}$ there are neither incoming nor outgoing coherent wavefronts centred on the particle, for $\phi_{\text {out }}$ both types of wavefronts are present ${ }^{17}$.

We saw this in the two examples from section 5, both of which described natural processes. For the second example, involving an external driving force, the retarded potential $\phi_{r}$ perfectly matched the total potential $\phi$, so the in potential $\phi_{\text {in }}$ vanished at all times. The advanced potential, however, did not match the total potential, which meant that the out potential was quite complicated. For example, before the force was applied there was no radiation present in the total field, but the out potential described incoming packets of radiation converging on the particle. These incoming packets were needed to cancel the incoming packets described by the advanced potential.

Similarly, for the first example the in field was simple, describing a single wavepacket propagating to the right, while the out field was complex, describing two wavepackets, one propagating to the right and one propagating to the left. The leftwardly-propagating wavepacket in the out field was needed to cancel part of the incoming radiation described by the advanced field.

In summary, we have seen that the equations of motion for the toy model are time-reversal invariant, but when the initial conditions for the model are chosen so as to correspond to the initial conditions of the actual world, the resulting radiative processes are time asymmetric. We called these natural processes, and found that in describing them it was convenient to reformulate the theory by expressing it in terms of time asymmetric retarded or advanced solutions. The resulting reformulations give useful descriptions of natural processes: the retarded formulation allows us to predict the future of a natural process based on an initial state, and the advanced formulation allows us to retrodict the past of a natural process based on a final state. The retarded and advanced formulations are both mathematically well defined, but because the time asymmetry of the retarded solutions matches the time asymmetry of the natural processes, the description of these processes is particularly simple in the retarded formulation. Although we have derived these results in the context of a simple toy model, this was only a pedagogical convenience. The physical principles involved are quite general: they

${ }^{17}$ Note that $\phi_{\text {out }}=\phi_{\text {in }}+\phi_{\text {rad }}$, where $\phi_{\text {rad }} \equiv \phi_{r}-\phi_{a}$ describes both incoming and outgoing coherent wavefronts centred on the particle. 
apply to many different systems in which waves can be radiated, and should help clarify how the radiative arrow of time justifies the use of the retarded solutions.

\section{References}

[1] Jackson J D 1975 Classical Electrodynamics 2nd edn (New York: Wiley)

[2] Popper K R 1956 Nature 177538

[3] Boozer A D 2007 Eur. J. Phys. 28 447-64

[4] Sakurai J J 1994 Modern Quantum Mechanics revised edn (Reading, MA: Addison-Wesley)

[5] Griffiths D J 1989 Introduction to Electrodynamics 2nd edn (Englewood Cliffs, NJ: Prentice Hall)

[6] Rohrlich F 1965 Classical Charged Particles: Foundations of Their Theory (Reading, MA: Addison-Wesley)

[7] Marion J B and Thornton S T 1988 Classical Dynamics of Particles and Systems 3rd edn (Fort Worth: Harcourt Brace Jovanovich)

[8] Huang K 1987 Statistical Mechanics 2nd edn (New York: Wiley) 